\title{
Masses and Dualities in Extended Freedman-Townsend Models
}

\author{
Ulrich Theis \\ Institute for Theoretical Physics \\ Friedrich-Schiller-University Jena \\ Max-Wien-Platz 1，D-07r43 Jena, Germany
}

\begin{abstract}
We consider some generalizations of Freedman-Townsend models of self-interacting antisymmetric tensors, involving couplings to further form fields introduced by Henneaux and Knaepen. We show how these fields can provide masses to the tensors by means of the Stückelberg mechanism and implement the latter in four-dimensional $\mathcal{N}=1$ superspace. The duality properties of the form fields are studied, and the paradoxical situation of a duality between a free and an interacting theory is encountered.
\end{abstract}

\section{INTRODUCTION}

In the effort to relate Calabi-Yau compactifications of ten-dimensional superstring theories to the real world, the method of turning on electric and magnetic fluxes of various $p$-form fields has gained a lot of attention due to the observation that the resulting scalar potentials in the low-energy effective supergravity theories can lift the vacuum degeneracy.

In four-dimensional models derived from such flux compactifications of Type II strings, massive antisymmetric tensor fields (2-forms) appear [1], whose properties until recently have not been investigated in much detail. This situation is quickly improving, however; in particular, the corresponding gauged $\mathcal{N}=2$ supergravity theories containing (multi-) tensor multiplets have been worked out in 2, 3], drawing on results obtained in [4].

The simultaneous introduction of electric and magnetic charges in this context still poses some problems. Recently, D'Auria et al. have suggested in [5] that a reformulation in terms of Freedman-Townsend (FT) models may provide a solution. These models describe $(n-2)$-forms in $n$ dimensions with non-polynomial interactions and are dual to non-linear sigma models of scalar fields [6, 7]. In flux compactifications of Type II strings, FT models can arise by dualization of the Ramond-Ramond scalars into tensors; the non-abelian nature of the (Heisenberg) algebra of isometries of the target space metric translates into FT-like interactions. The question then arose in [5] how to couple the self-interacting tensors to vector fields in a way which corresponds to turning on RR fluxes. Magnetic charges in particular should introduce mass terms for the tensors in a gauge invariant manner. This was achieved for the Neveu-Schwarz 2-form by means of the Stückelberg mechanism, but not for the remaining RR tensors. In this letter we close this gap by deriving the most general massive FT model.

Toward this end, we employ an extension of FT models constructed by Henneaux and Knaepen in [8], which involves couplings of the $(n-2)$-forms to $p$-forms of lower rank. These additional fields will provide the degrees of freedom that turn the tensors into massive fields. Gauge invariance is maintained through the Stückelberg mechanism as in [5]. This is different from the methods already described by Freedman and Townsend [7], where either gauge invariance was broken by the mass terms, or where through a coupling to Yang-Mills fields the latter were made massive.

Our purely bosonic models can be generalized to $\mathcal{N}=1$ supersymmetric models using the superfield approach of [9], as we demonstrate in the last section of this letter. A massive $\mathcal{N}=1$ tensor multiplet was first derived in 10], and has recently been formulated in 11] (see also 12, 13, 14]) using the Stückelberg mechanism. Here we generalize the latter construction to an arbitrary number of tensor multiplets with self-interactions of the FT-type. Before we turn to the construction of massive FT models, we first review the Henneaux-Knaepen (HK) extensions and make some interesting observations about their duality properties, which to our knowledge have not been presented in the literature so far.

\section{DUALITIES IN EXTENDED FT MODELS}

Consider sets of $(n-2)$-forms $B_{I}, p$-forms $A^{a}$ with $p<$ $n-2$, and auxiliary 1 -forms $V^{I}$ in $n>2$ dimensions. The HK action in first-order form reads [8] (where we write $A^{a}$ as a column vector and wedge products are understood)

$$
\begin{array}{r}
S_{1}=\frac{1}{2} \int\left(\Delta_{I J} * V^{I} V^{J}+2 B_{I} G^{I}\right. \\
\left.+* \mathcal{F}^{t} M \mathcal{F}+\mathcal{F}^{t} N \mathcal{F}\right),
\end{array}
$$

with field strengths

$$
\begin{aligned}
G^{I} & =\mathrm{d} V^{I}+\frac{1}{2} f_{J K}{ }^{I} V^{J} V^{K} \\
\mathcal{F} & =\left(\mathrm{d}+V^{I} T_{I}\right) A .
\end{aligned}
$$

The $f_{I J}{ }^{K}$ are structure constants of some arbitrary Lie algebra $\mathcal{G}$, while the real matrices $T_{I}{ }^{a}{ }_{b}$ define a representation of $\mathcal{G}$,

$$
f_{[I J}^{L} f_{K] L}^{M}=0, \quad\left[T_{I}, T_{J}\right]=f_{I J}^{K} T_{K} .
$$

$\Delta$ and $M$ are arbitrary positive-definite symmetric matrices. The topological term proportional to the matrix 
$N=(-)^{p+1} N^{t}$ can be present only if $2(p+1)=n$. It was not part of the original formulation of HK models, but should be included as it arises naturally in supersymmetric extensions [9]. $\Delta, M$, and $N$ are neither required to be $\mathcal{G}$-invariant, nor do they have to be constant and can depend on further fields.

$S_{1}$ is invariant under abelian gauge transformations $\delta=$ $\delta_{\lambda}+\delta_{C}$ with

$$
\begin{aligned}
\delta A & =\left(\mathrm{d}+V^{I} T_{I}\right) \lambda \Rightarrow \delta \mathcal{F}=G^{I} T_{I} \lambda \\
\delta B_{I} & =\mathrm{d} C_{I}+f_{I J}{ }^{K} V^{J} C_{K}-\left(* \mathcal{F}^{t} M+\mathcal{F}^{t} N\right) T_{I} \lambda \\
\delta V^{I} & =0
\end{aligned}
$$

where $\lambda^{a}$ and $C_{I}$ are arbitrary $(p-1)$ - and $(n-3)$-forms, respectively. Invariance under $\delta_{\lambda}$ is obvious, while invariance under $\delta_{C}$ (modulo a boundary term) follows from the identity $\mathrm{d} G^{I}+f_{J K}{ }^{I} V^{J} G^{K}=0$. Note that, despite of the similarity of $G^{I}$ to a Yang-Mills field strength, there is no gauge transformation associated with $V^{I}$.

The second-order version is obtained by eliminating $V^{I}$ via their algebraic equations of motion, which can be brought into the form

$$
\begin{aligned}
& {\left[\left(\Delta_{I J}+A^{t} M_{I J} i_{A}\right) *-f_{I J}{ }^{K} B_{K}-(-)^{p} A^{t} N_{I J} A\right] V^{J}=} \\
& =(-)^{n} \mathrm{~d} B_{I}-(-)^{p}\left(* F^{t} M+F^{t} N\right) T_{I} A
\end{aligned}
$$

where $F=\mathrm{d} A$. Here we have introduced the matrices

$$
M_{I J}=T_{I}^{t} M T_{J}, \quad N_{I J}=T_{I}^{t} N T_{J},
$$

and the operator

$$
i_{A_{p}}=\frac{1}{p !} A^{\mu_{1} \ldots \mu_{p}} \frac{\partial^{p}}{\partial \mathrm{d} x^{\mu_{p}} \ldots \partial \mathrm{d} x^{\mu_{1}}},
$$

which arises in the above equation by virtue of the identity $*\left(\omega_{q} A_{p}\right)=(-)^{p(n-1)} i_{A_{p}} * \omega_{q}$. In general, (8) can be solved for $V^{I}$ only perturbatively, the solution being nonpolynomial in both $B_{I}$ and $A^{a}$. Some examples of exact solutions (involving one $B$ and one $A$ ) can be found in 9, 15, 16]. Note that when $N$-terms are present, they give rise to couplings of $\mathrm{d} B_{I}$ to abelian Chern-Simons forms of $A^{a}$.

To derive the dual formulation one solves the equations of motion of $B_{I}$, namely the flatness conditions $G^{I}=0$, in terms of scalars $\phi^{I}$ :

$$
V^{I} T_{I}=\mathrm{e}^{-\phi \cdot T} \mathrm{de}^{\phi \cdot T}=\mathrm{d} \phi^{I} E_{I}^{J}(\phi) T_{J} .
$$

The vielbein $E_{I}^{J}$ can be expressed as

$$
E_{I}^{J}(\phi)=\int_{0}^{1} \mathrm{~d} t\left(\mathrm{e}^{t \phi \cdot f}\right)_{I}^{J}, \quad(\phi \cdot f)_{I}^{J}=\phi^{K} f_{I K}{ }^{J} .
$$

When inserted into $\mathcal{F}$, a local field redefinition allows to absorb the flat connections $V^{I}(\phi)$,

$$
\mathcal{F}=\mathrm{e}^{-\phi \cdot T} \hat{F}, \quad \hat{F}=\mathrm{d} \hat{A}, \quad \hat{A}=\mathrm{e}^{\phi \cdot T} A .
$$

The dual action in terms of the scalars $\phi^{I}$ and the redefined $p$-forms $\hat{A}$ then reads

$$
S_{2}=\frac{1}{2} \int\left(g_{I J} * \mathrm{~d} \phi^{I} \mathrm{~d} \phi^{J}+* \hat{F}^{t} \hat{M} \hat{F}+\hat{F}^{t} \hat{N} \hat{F}\right) .
$$

Here the target space metric of the NLSM is given by

$$
g_{I J}(\phi)=E_{I}^{K}(\phi) E_{J}^{L}(\phi) \Delta_{K L},
$$

while the gauge coupling functions are

$$
\hat{M}(\phi)=\mathrm{e}^{-\phi \cdot T^{t}} M \mathrm{e}^{-\phi \cdot T}, \quad \hat{N}(\phi)=\mathrm{e}^{-\phi \cdot T^{t}} N \mathrm{e}^{-\phi \cdot T} .
$$

It is now obvious (although it was not observed in [8]) that also the $p$-forms can be dualized, since the $\hat{A}$ enter the action only via their derivatives. By adding to $S_{2}$ a term $\mathrm{d} \hat{A}_{\tilde{p}}^{t} \hat{F}$ (where $\tilde{p}=n-2-p$ ) and integrating out $\hat{F}$, we arrive at the dual action for the $\tilde{p}$-forms $\hat{A}_{\tilde{p}}$. In the process, the matrices $\hat{M}$ and $\hat{N}$ get inverted. From (16) it is clear that this effectively interchanges $T_{I}$ and $-T_{I}{ }^{t}$. We can now reverse the whole procedure; a redefinition $\hat{A}_{\tilde{p}}=\mathrm{e}^{-\phi \cdot T^{t}} A_{\tilde{p}}$ reintroduces flat connections $V^{I}(\phi)$, which can be promoted to unconstrained fields by enforcing the flatness conditions $G^{I}=0$ through Lagrange multipliers $B_{I}$. This results in a first-order action of the form (11) for $B_{I}$ and $A_{\tilde{p} a}$. We conclude that HK models with $p$-forms and $\tilde{p}$-forms, respectively, are dual to each other upon the substitution

$$
\begin{aligned}
T_{I} & \leftrightarrow-T_{I}^{t} \\
M & \leftrightarrow\left[M-(-)^{p} N M^{-1} N\right]^{-1} \\
N & \leftrightarrow(-)^{p}\left[N-(-)^{p} M N^{-1} M\right]^{-1} .
\end{aligned}
$$

Note that the matrices $-T_{I}^{t}$ form a representation of the Lie algebra $\mathcal{G}$.

Let us repeat the steps we have taken: First dualize the $B_{I}$ into $\phi^{I}$, then redefine $A_{p} \rightarrow \hat{A}_{p}$, dualize these $\hat{A}_{p}$ into $\hat{A}_{\tilde{p}}$, and after another redefinition $\hat{A}_{\tilde{p}} \rightarrow A_{\tilde{p}}$ dualize the $\phi^{I}$ back into $B_{I}$. This raises the question whether there is a more direct dualization from one HK model to the other.

A puzzling observation is that if $\left(M T_{I}\right)^{t}=-M T_{I}$ and $\left(N T_{I}\right)^{t}=(-)^{p} N T_{I}$ for all $I$ (for example antisymmetric $T_{I}$ in the case $M=\mathbb{1}$ and $N=0$, which corresponds to the original HK models), then $\hat{M}=M, \hat{N}=N$, and the $\hat{A}^{a}$ decouple from the $\phi^{I}$,

$$
S_{2}=\frac{1}{2} \int\left(g_{I J} * \mathrm{~d} \phi^{I} \mathrm{~d} \phi^{J}+* \hat{F}^{t} M \hat{F}+\hat{F}^{t} N \hat{F}\right) .
$$

If one now dualizes the $\phi^{I}$ back into tensors $B_{I}$, no interactions between the latter and $\hat{A}^{a}$ arise. Indeed, (18) can be obtained from a first-order action without couplings of $\hat{A}^{a}$ to $V^{I}$,

$$
\begin{array}{r}
S_{1}=\frac{1}{2} \int\left(\Delta_{I J} * V^{I} V^{J}+2 B_{I} G^{I}\right. \\
\left.+* \hat{F}^{t} M \hat{F}+\hat{F}^{t} N \hat{F}\right),
\end{array}
$$


hence after elimination of $V^{I}$ the $B_{I}$ and $\hat{A}^{a}$ do not interact.

This seems to provide a duality between an interacting theory (coupling fields $B_{I}$ and $A^{a}$ ) and a free theory (with decoupled fields $\phi^{I}$ and $\hat{A}^{a}$ ), disregarding selfinteractions of the $B_{I}$ and $\phi^{I}$, respectively, for the sake of argument ${ }^{1}$. We are not aware of a resolution of this paradox. That the tensor side of the duality really involves genuine interactions between $B_{I}$ and $A^{a}$ should be ensured by the fact that their couplings cannot be removed by any local field redefinition. This was proved in [17] by BRST cohomological methods for any representation of $\mathcal{G}$. The standard duality procedure we have employed is a non-local operation, of course, and under the specific circumstances spelled out above it can be followed with a local field redefintion such that the combination apparently amounts to a non-local field redefinition on the other side of the duality which removes the interactions.

\section{MASSIVE FT MODELS}

Let us now specialize to the case $p=n-3$. Then it is possible to introduce masses for the tensors $B_{I}$ of pure FT models by means of the Stückelberg mechanism, where the $p$-forms $A^{a}$ serve as compensators. Toward this end we introduce constant mass parameters $m^{a I}$ and another set of parameters $n_{I a}(m)$, which are assumed to satisfy the orthonormality condition

$$
n_{I a} m^{a J}=\delta_{I}^{J}
$$

Note that one needs at least as many $p$-forms as there are tensors, $\left(n_{A}=\delta_{a}^{a}\right) \geq\left(n_{B}=\delta_{I}^{I}\right)$. Moreover, if $n_{A}>n_{B}$, the $n_{I a}$ are in general not uniquely defined. Condition (20) guarantees that the matrices

$$
T_{I}^{a}{ }_{b}=m^{a J} f_{J I}{ }^{K} n_{K b}
$$

form a representation of the Lie algebra $\mathcal{G}$ with structure constants $f_{I J}{ }^{K}$. The gauge transformations

$$
\begin{gathered}
\delta A^{a}=\mathrm{d} \lambda^{a}+m^{a I} C_{I}, \quad \delta V^{I}=0 \\
\delta B_{I}=\mathrm{d} C_{I}+f_{I J}{ }^{K} V^{J}\left(C_{K}+n_{K a} \mathrm{~d} \lambda^{a}\right)
\end{gathered}
$$

leave invariant the combination $\mathcal{F}^{a}-m^{a I} B_{I}$ with $\mathcal{F}^{a}$ as in (3) and $T_{I}$ as in (21),

$$
\delta \mathcal{F}^{a}=m^{a I} \delta B_{I}
$$

Since the tensor transformations (23) are of the form (6) with $C_{I}=n_{I a} \delta A^{a}$ and $\lambda^{a}=0$, it immediately follows

\footnotetext{
${ }^{1}$ In the simplest setup where this phenomenon occurs, namely one $B$ and two $A^{a}$ with $T^{a}{ }_{b}=\varepsilon_{a b}, M=1, N=0$, there are no interactions at all on the scalar side of the duality.
}

that the first-order action

$$
\begin{aligned}
S_{1}=\frac{1}{2} \int[ & \Delta_{I J} * V^{I} V^{J}+2 B_{I} G^{I} \\
& +M_{a b} *\left(\mathcal{F}^{a}-m^{a I} B_{I}\right)\left(\mathcal{F}^{b}-m^{b J} B_{J}\right) \\
& \left.+N_{a b}\left(\mathcal{F}^{a}-m^{a I} B_{I}\right)\left(\mathcal{F}^{b}-m^{b J} B_{J}\right)\right]
\end{aligned}
$$

is gauge invariant. The topological terms in the last line are now only possible if $n=4$ and $p=1$, which is just what we need in applications to flux compactifications as in [5]. The auxiliary fields $V^{I}$ can again be eliminated; their equations of motion are obtained from (8) by replacing $F^{a}$ with $F^{a}-m^{a I} B_{I}$ and $T_{I}$ with (21).

Since $S_{1}$ depends on the inverse mass parameters $n_{I a}$ through the mass ratios $T_{I}$, taking the massless limit is subtle: The projector

$$
P_{b}^{a}=\delta_{b}^{a}-m^{a I} n_{I b}
$$

has $n_{B}$ eigenvectors $m^{a I}$ with zero eigenvalue, and we can use the freedom in solving (20) for $n_{I a}$ to choose the latter such that $P$ has $n_{B}$ vanishing rows. With this choice, we introduce rescaled fields

$$
\begin{gathered}
\mathcal{A}_{I}=n_{I a} A^{a}, \quad \mathcal{A}^{a}=P^{a}{ }_{b} A^{b} \\
\Leftrightarrow \quad A^{a}=m^{a I} \mathcal{A}_{I}+\mathcal{A}^{a} .
\end{gathered}
$$

$\mathcal{A}^{a}$ has $n_{A}-n_{B}$ non-vanishing components. When expressed in terms of these fields, the inverse mass parameters disappear from the action due to $T_{I}{ }^{a}{ }_{b} A^{b}=$ $m^{a J} f_{J I}{ }^{K} \mathcal{A}_{K}$, and the massless limit can be safely taken. The $\mathcal{A}_{I}$ are precisely the compensators that are eaten by $B_{I}$ in the "unitary gauge" where the $\mathcal{A}_{I}$ are transformed away using (22).

Note that if we define "electric charges" $e_{a}{ }^{I}=N_{a b}^{(0)} m^{b I}$, where the superscript (0) refers to the constant part of $N_{a b}$, then

$$
m^{a I} e_{a}^{J}-m^{a J} e_{a}^{I}=0
$$

trivially by virtue of the symmetry of $N_{a b}^{(0)}$.

The mass matrix for the $B_{I}$ is complicated in general. To determine it we can drop all interactions, which amounts to setting $f_{I J}{ }^{K}=0$ and $\Delta_{I I}^{(0)} V^{J}=* \mathrm{~d} B_{I}$ in $S_{1}$. We then follow D'Auria and Ferrara [12] in deriving the linearized equations of motion for the fields

$$
b_{I}=\left(B-\mathcal{M}^{-1} \mathcal{N} * B\right)_{I},
$$

which are transversal on-shell, $\mathrm{d} * b_{I}=0$. Here we have defined

$$
\mathcal{M}^{I J}=m^{a I} M_{a b}^{(0)} m^{b J}, \quad \mathcal{N}^{I J}=m^{a I} e_{a}{ }^{J}
$$

and assumed that $\mathcal{M}$ is invertible. A short calculation yields the equations

$$
\left[* \mathrm{~d} * \mathrm{~d}-\left(\mathbb{1}+\mathcal{M}^{-1} \mathcal{N} \mathcal{M}^{-1} \mathcal{N}\right) \Delta^{(0)} \mathcal{M}\right]_{I}^{J} b_{J}=0,
$$


from which we can read off the mass matrix. As is familiar from flux compactifications, it receives contributions from the electric charges.

The tensors $B_{I}$ could be eliminated instead of the $V^{I}$, which then gives rise to a theory of massive vectors.

\section{MASSIVE FT MODELS IN SUPERSPACE}

Just like the massless FT models 18, 19, 20 and their HK extensions [9], the massive four-dimensional FT models can be formulated in $\mathcal{N}=1$ superspace. Following 9 , we embed the tensors $B_{I}$ in chiral spinor superfields $\Psi_{\alpha I}$ and the vectors $A^{a}, V^{I}$ in real scalar superfields, for which we shall use the same labels. For the latter, we construct super-field strengths

$$
\begin{aligned}
Y_{\alpha}^{a} & =-\frac{\mathrm{i}}{4} \bar{D}^{2}\left[\mathrm{e}^{-2 \mathrm{i} V \cdot T} D_{\alpha}\left(\mathrm{e}^{\mathrm{i} V \cdot T} A\right)\right]^{a} \\
W_{\alpha}^{I} T_{I} & =-\frac{\mathrm{i}}{4} \bar{D}^{2}\left(\mathrm{e}^{-2 \mathrm{i} V \cdot T} D_{\alpha} \mathrm{e}^{2 \mathrm{i} V \cdot T}\right),
\end{aligned}
$$

with $T_{I}$ as in (21). These being chiral, the first-order action

$$
\begin{aligned}
S_{1}=\int \mathrm{d}^{4} x \mathrm{~d}^{2} \theta[ & k_{a b}\left(Y^{a}-m^{a I} \Psi_{I}\right)\left(Y^{b}-m^{b J} \Psi_{J}\right) \\
& \left.+W^{I} \Psi_{I}+\mathrm{d}^{2} \bar{\theta} h(V)\right]+ \text { c.c. },
\end{aligned}
$$

where $k_{a b}=k_{b a}$ may depend on further chiral superfields, is manifestly supersymmetric. The function $h(V)$ need not be quadratic in $V^{I}$, as long as the latter can be eliminated.

The nontrivial part is to verify gauge invariance. Let us start with the simpler tensor gauge transformations; embedding the parameters $C_{I}$ into real scalar superfields of the same name, these read

$$
\begin{gathered}
\delta_{C} A^{a}=m^{a I} C_{I}, \quad \delta_{C} V^{I}=0 \\
\delta_{C} \Psi_{\alpha I}=-\frac{\mathrm{i}}{4} \bar{D}^{2}\left[\mathrm{e}^{-2 \mathrm{i} V \cdot f} D_{\alpha}\left(\mathrm{e}^{\mathrm{i} V \cdot f} C\right)\right]_{I} .
\end{gathered}
$$

Here we use $(V \cdot f)_{I}^{J}=V^{K} f_{I K}{ }^{J}$. To compute the induced transformations of $Y^{a}$, we need the identity

$$
\left(\mathrm{e}^{\mathrm{i} V \cdot T}\right)_{b}^{a}=P_{b}^{a}+m^{a I}\left(\mathrm{e}^{\mathrm{i} V \cdot f}\right)_{I}^{J} n_{J b},
$$

which follows from $(V \cdot T)^{a}{ }_{b}=m^{a I}(V \cdot f)_{I}{ }^{J} n_{J b}$ and (20) $(P$ was defined in (26) $)$. It is now easy to show that $\delta_{C} Y^{a}=m^{a I} \delta_{C} \Psi_{I}$, which implies invariance of the first line in (34); just move $m^{a I}$ in $\delta_{C} A^{a}$ past the $\mathrm{e}^{\mathrm{i} V \cdot T}$ 's in $Y^{a}$, which get converted into $\mathrm{e}^{\mathrm{i} V \cdot f}$ 's. A proof of invariance of the second line can be found in section 3 of [9] and relies on the Bianchi identity satisfied by $W^{I}$, which are standard super-gauge field strengths,

$$
D^{\alpha}\left(W_{\alpha} \mathrm{e}^{-2 \mathrm{i} V \cdot f}\right)^{I}\left(\mathrm{e}^{\mathrm{i} V \cdot f}\right)_{I}^{J}-\text { c.c. }=0 .
$$

The vector gauge transformations generalize to

$$
\begin{gathered}
\delta_{\Lambda} A^{a}=\Lambda^{a}+\bar{\Lambda}^{a}, \quad \delta_{\Lambda} V^{I}=0 \\
\delta_{\Lambda} \Psi_{\alpha I}=-\frac{\mathrm{i}}{4} \bar{D}^{2}\left[\mathrm{e}^{-2 \mathrm{i} V \cdot f} D_{\alpha}\left(\mathrm{e}^{\mathrm{i} V \cdot f} n_{a} \delta_{\Lambda} A^{a}\right)\right]_{I},
\end{gathered}
$$

where $\Lambda^{a}$ are arbitrary chiral superfields. For $\Psi_{I}$, these have the same form as a tensor gauge transformation $\delta_{C}$, so the second line in (34) is invariant. Using the identity (37), the transformations of $Y^{a}$ turn into

$$
\delta_{\Lambda} Y_{\alpha}^{a}=-\frac{\mathrm{i}}{4} \bar{D}^{2}\left[\mathrm{e}^{-2 \mathrm{i} V \cdot T} P D_{\alpha} \Lambda\right]^{a}+m^{a I} \delta_{\Lambda} \Psi_{\alpha I} .
$$

By multiplying (37) from the right with $P$, one shows that $\mathrm{e}^{-2 \mathrm{i} V \cdot T} P=P$. The chirality of $\Lambda^{a}$ then implies that $\bar{D}^{2} D_{\alpha} \Lambda^{a}=0$, so we find again $\delta_{\Lambda} Y^{a}=m^{a I} \delta_{\Lambda} \Psi_{I}$, which proves invariance of the action.

In the free case, $f_{I J}{ }^{K}=0$, it is possible to add a term

$$
\int \mathrm{d}^{4} x \mathrm{~d}^{2} \theta \hat{e}_{a}^{I} \Psi_{I}\left(Y^{a}-\frac{1}{2} m^{a J} \Psi_{J}\right)+\text { c.c. }
$$

to the action (34), as was observed in [11]. Gauge invariance constrains the charges $\hat{e}_{a}^{I}$ to satisfy (28). In the presence of interactions, where $f_{I J}{ }^{K} \neq 0$, this term is not invariant anymore under the above gauge transformations. Unfortunately, we have not been able to find a suitable modification of the action and transformations to restore invariance.

I would like to thank Friedemann Brandt, Bernard de Wit and Stefan Vandoren for stimulating discussions about dualities. Thanks also to Mario Trigiante for making me think about massive Freedman-Townsend models. Supported by the DFG within the priority program SPP 1096 on string theory.

* Electronic address: Ulrich.Theis@uni-jena.de

[1] J. Louis and A. Micu, Type II theories compactified on Calabi-Yau threefolds in the presence of background fluxes. Nucl. Phys. B635 (2002) 395, hep-th/0202168.

[2] R. D'Auria, G. Dall'Agata, L. Sommovigo and S. Vaula, $D=4, N=2$ gauged supergravity in the presence of tensor multiplets. Nucl. Phys. B682 (2004) 243, hep-th/0312210.

[3] R. D'Auria, L. Sommovigo and S. Vaula, $N=2$ supergravity Lagrangian coupled to tensor multiplets with electric and magnetic fluxes. J. High Energy Phys. 11 (2004) 028, hep-th/0409097.

[4] U. Theis and S. Vandoren, $N=2$ supersymmetric scalar-tensor couplings. J. High Energy Phys. 04 (2003) 042, hep-th/0303048.

[5] R. D'Auria, S. Ferrara, M. Trigiante and S. Vaula, Scalar potential for the gauged Heisenberg algebra and a non-polynomial antisymmetric tensor theory. Phys. Lett. B610 (2005) 270, hep-th/0412063.

[6] V.I. Ogievetsky and I.V. Polubarinov, The notoph and its possible interactions. Yad. Fiz. 4 (1966) 216, Sov. J. Nucl. Phys. 4 (1967) 156 
[7] D. Freedman and P.K. Townsend, Antisymmetric tensor gauge theories and nonlinear sigma models. Nucl. Phys. B177 (1981) 282.

[8] M. Henneaux and B. Knaepen, All consistent interactions for exterior form gauge fields. Phys. Rev. D56 (1997) 6076, hep-th/9706119.

[9] F. Brandt and U. Theis, $D=4, N=1$ supersymmetric Henneaux-Knaepen models. Nucl. Phys. B550 (1999) 495, hep-th/9811180.

[10] W. Siegel, Gauge spinor superfield as a scalar multiplet. Phys. Lett. B85 (1979) 333.

[11] T.W. Grimm and J. Louis, The effective action of $N=1$ Calabi-Yau orientifolds. Nucl. Phys. B699 (2004) 387, hep-th/0403067.

[12] R. D'Auria and S. Ferrara, Dyonic masses from conformal field strengths in $D$ even dimensions. Phys. Lett. B606 (2005) 211, hep-th/0410051.

[13] J. Louis and W. Schulgin, Massive tensor multiplets in $N=1$ supersymmetry. Fortschr. Phys. 53 (2005) 235, hep-th/0410149.

[14] S.M. Kuzenko, On massive tensor multiplets. J. High Energy Phys. 01 (2005) 041, hep-th/0412190.
[15] F. Brandt and N. Dragon, Nonpolynomial gauge invariant interactions of 1-form and 2-form gauge potentials. In: Theory of Elementary Particles (Proc. Int. Symp. Ahrenshoop, Buckow 1997), Wiley-VCH 1998, 149. hep-th/9709021.

[16] U. Theis, New N=2 supersymmetric vector-tensor interaction. Phys. Lett. B486 (2000) 443, hep-th/0005044.

[17] M. Henneaux and B. Knaepen, A theorem on first-order interaction vertices for free p-form gauge fields. Int. J. Mod. Phys. A15 (2000) 3535, hep-th/9912052.

[18] U. Lindström and M. Roček, Scalar tensor duality and $N=1,2$ non-linear $\sigma$-models. Nucl. Phys. B222 (1983) 285.

[19] T.E. Clark, C.-H. Lee and S.T. Love, Supersymmetric tensor gauge theories. Mod. Phys. Lett. A4 (1989) 1343.

[20] K. Furuta, T. Inami, H. Nakajima and M. Nitta, Supersymmetic extension of the non-abelian scalar-tensor duality. Prog. Theor. Phys. 106 (2001) 851, hep-th/0106183. 